\title{
A BOUND FOR THE DISTANCE BETWEEN FRACTIONAL BROWNIAN MOTION AND THE SPACE OF GAUSSIAN MARTINGALES ON AN INTERVAL
}

UDC 519.21

\author{
O. L. BANNA AND YU. S. MISHURA
}

\begin{abstract}
We obtain a lower bound for the distance between fractional Brownian motion and the space of Gaussian martingales on an interval. The distances between fractional Brownian motion and some subspaces of Gaussian martingales are compared. The upper and lower bounds are obtained for the constant in the representation of a fractional Brownian motion in terms of the Wiener process.
\end{abstract}

\section{INTRODUCTION}

A zero mean Gaussian process $\left\{B_{t}^{H}, t \geq 0\right\}, B_{0}^{H}=0$, with the covariance function

$$
\mathrm{E} B_{t}^{H} B_{s}^{H}=\frac{1}{2}\left(t^{2 H}+s^{2 H}-|t-s|^{2 H}\right)
$$

is called a fractional Brownian motion with the Hurst index $H \in(0,1)$. In what follows we consider the case where $H \in\left(\frac{1}{2}, 1\right)$. It is known that the fractional Brownian motion $\left\{B_{t}^{H}, t \in[0, T]\right\}$ admits the representation

$$
B_{t}^{H}=\int_{0}^{t} z(t, s) d W_{s}
$$

where $\left\{W_{t}, t \in[0, T]\right\}$ is a Wiener process,

$$
\begin{gathered}
z(t, s)=\left(H-\frac{1}{2}\right) c_{H} s^{1 / 2-H} \int_{s}^{t} u^{H-1 / 2}(u-s)^{H-3 / 2} d u, \\
c_{H}=\left(\frac{2 H \cdot \Gamma\left(\frac{3}{2}-H\right)}{\Gamma\left(H+\frac{1}{2}\right) \Gamma(2-2 H)}\right)^{1 / 2},
\end{gathered}
$$

and where $\Gamma(x), x>0$, is the Gamma function (see [7]). Throughout the paper we use the notation $\alpha=H-\frac{1}{2}$.

The best approximation in the space $L_{\infty}\left([0, T] ; L_{2}(\Omega)\right)$ of fractional Brownian motion by martingales of the form

$$
\int_{0}^{t} a(s) d W_{s}
$$

is found in the papers [2]-4] and [6], where $W$ is a Wiener process and $a$ is a function possessing one of the following properties:

1) $a(s)$ is a constant, that is, $a(s)=a, s \in[0, T]$;

2010 Mathematics Subject Classification. Primary 60G15; Secondary 60G44.

Key words and phrases. Wiener process, fractional Brownian motion, Gaussian martingale, approximation in a class of functions. 
2) $a(s)$ is a power function of the form $a(s)=k \cdot s^{\alpha}$, where $k \in \mathbb{R}, \alpha=H-1 / 2$, and $H$ is the Hurst index of the fractional Brownian motion;

3) $a(s)$ is a power function of the form $a(s)=k \cdot s^{\gamma}$, where $k \in \mathbb{R}$ and $\gamma>0$;

4) $a(s)$ is a power function with a negative index, namely $a(s)=k \cdot s^{-\alpha}$, where $k>0, \alpha=H-1 / 2$, and $H$ is the Hurst index of the fractional Brownian motion;

5) $a(s)$ is a function of the form $a(s)=k_{1}+k_{2} \cdot s^{\alpha}$, where $k_{1}, k_{2} \in \mathbb{R}$ and $s \in[0, T]$.

For each case listed above, a function is known at which the square of the distance

$$
\rho_{T}:=\min _{a \in L_{2}[0, T]} \max _{0 \leq t \leq T} \mathrm{E}\left(B_{t}^{H}-\int_{0}^{t} a(s) d W_{s}\right)^{2}
$$

is minimal; the precise value of the minimum is evaluated in [2]-4] and [6]. To be specific, below we write the corresponding functions and minimal values for all five cases listed above.

1)

$$
\min _{a} \max _{0 \leq t \leq T} \mathrm{E}\left(B_{t}^{H}-M_{t}\right)^{2}=T^{2 H}\left(1-c_{1}^{2}\right)
$$

where

$$
c_{1}=c_{1}(H)=\alpha c_{H} \cdot \frac{1}{\alpha+1} \cdot B(1-\alpha, \alpha)
$$

(see [3]). In this case, $a_{\min }=c_{1}(H) \cdot T^{\alpha}$.

2)

$$
\min _{k} \max _{0 \leq t \leq T} \mathrm{E}\left(B_{t}^{H}-M_{t}\right)^{2}=T^{2 H}\left(1-\frac{c_{H}^{2}}{2 H}\right) .
$$

In this case, $k_{\min }=c_{H}($ see $[3])$.

3)

$$
\min _{\gamma} \min _{k} \max _{0 \leq t \leq T} \mathrm{E}\left(B_{t}^{H}-M_{t}\right)^{2}=T^{2 H}\left(1-c_{1}^{2}(H)\right) .
$$

4)

In this case, the minimum is attained for $\gamma=0$ and $k_{\min }=c_{1}(H) \cdot T^{\alpha}$ (see [3]).

$$
\min _{k} \max _{0 \leq t \leq T} \mathrm{E}\left(B_{t}^{H}-M_{t}\right)^{2}=\varphi_{1}\left(k^{*}\right) .
$$

The minimum is attained at the function $a(s)=k^{*} s^{1 / 2-H}$, where the coefficient $k^{*}$ is the minimum argument of two intersection points of the functions

$$
\varphi_{1}(k)=k^{\frac{2 H}{2 H-1}} c_{1}(H) \quad \text { and } \quad \varphi_{2}(k)=T^{2 H}-\frac{4 k H}{c_{H}} T+k^{2} \frac{T^{2-2 H}}{2-2 H}
$$

(see [4]); here

$$
c_{1}(H)=(p(H))^{-\frac{2 H}{2 H-1}} \frac{2 H-1}{1-H}\left(\frac{2 H}{c_{H}} p(H)-1\right) .
$$

$5)$

$$
\begin{aligned}
\min _{k_{1}, k_{2}} \max _{0 \leq t \leq T} \mathrm{E}\left(B_{t}^{H}-M_{t}\right)^{2} \\
\quad=T^{2 \alpha+1}-T^{2 \alpha+1} c_{H}^{2}\left(B^{2}(1-\alpha, \alpha)-\frac{2 B(1-\alpha, \alpha)}{\alpha}+\frac{(\alpha+1)^{2}}{\alpha^{2}(2 \alpha+1)}\right) .
\end{aligned}
$$

The minimum is attained for $k_{1}=k_{1}^{*}$ and $k_{2}=k_{2}^{*}$ (the precise values of the constants are given in [2] and [6]). 
In this paper, we obtain a nonzero lower bound for $\rho_{T}$.

It is proved in the paper [3] that the best approximation among the functions possessing the properties 1)-3) above is given by the constant function mentioned in the case 1). Below, we compare the minimal values for the cases 1) and 5), as well.

The paper is organized as follows. In Section 2, we recall the properties of a function that minimizes the square of the distance $\rho_{T}$ under the assumption that such a function exists (the explicit form of this function as well as conditions for its existence are not yet known). A lower bound for the best approximation of the fractional Brownian motion by Gaussian martingales is obtained in Section [3. In Section 4, we compare the best approximations of a fractional Brownian motion by corresponding Gaussian martingales in some classes of functions. A new upper bound for the square of the distance $\rho_{T}$ as well as a new estimate for the constant $c_{H}$ are obtained in Section [5 these results are based on the properties established in Section 2. Various bounds for the constant $c_{H}$ are compared numerically and graphically in Section 6.

\section{Auxiliary Results}

Let $\left\{W_{t}, t \in[0, T]\right\}$ be a Wiener process and let $B_{t}^{H}=\int_{0}^{t} z(t, s) d W_{s}$ be a fractional Brownian motion with the Hurst index $H>1 / 2$. Set $\alpha=H-1 / 2$. For a locally square integrable function $a: \mathbb{R}_{+} \rightarrow \mathbb{R}$, let

$$
F_{a}(t)=\mathrm{E}\left[\left(B_{t}^{H}-\int_{0}^{t} a(s) d W_{s}\right)^{2}\right]=\int_{0}^{t}(a(s)-z(t, s))^{2} d s .
$$

If $c>0$ is arbitrary, then

$$
F_{a}(t)=c^{2 H} F_{c^{-\alpha} a(c \cdot)}(t / c) .
$$

This result follows from the equality of distributions

$$
\left(c^{-1 / 2} W_{c t}, c^{-H} B_{c t}^{H}\right) \stackrel{d}{=}\left(W_{t}, B_{t}^{H}\right) .
$$

Consider the optimization problem

$$
M_{a}:=\max _{t \in[0,1]} F_{a}(t) \rightarrow \min
$$

with respect to the function $a$.

Let a function $a^{*} \in C\left(\mathbb{R}_{+}\right)$minimize the functional $M_{a}$.

Lemma 2.1. $M_{a^{*}}=F_{a^{*}}(1)$.

Proof. Assume that $F_{a^{*}}(1)<M_{a^{*}}$. Since $F_{a^{*}}(t)$ is continuous with respect to $t$, a number $c>1$ exists such that $F_{a^{*}}(t)<M_{a^{*}}$ for $t \in[1, c]$. Thus $\max _{t \in[0, c]} F_{a^{*}}(t)=M_{a^{*}}$. Put $b(t)=c^{-\alpha} a^{*}(t c)$. Equality (2.1) implies that $F_{b}(t)=c^{-2 H} F_{a^{*}}(t c), t \in[0,1]$. This yields $M_{b}=c^{-2 H} M_{a^{*}}<M_{a^{*}}$ which contradicts the assumption.

Now we assume that the function $F_{a^{*}}(t)$ is continuously differentiable.

Lemma 2.2. $F_{a^{*}}^{\prime}(1) \geq 2 H F_{a^{*}}(1)$.

Proof. The preceding lemma implies that $F_{a^{*}}^{\prime}(1) \geq 0$. For $c>1$, let $b(t)=c^{-\alpha} a^{*}(t c)$, $t \in[0,1]$. Equality (2.1) implies $F_{b}(t)=c^{-2 H} F_{a}(t c), t \in[0,1 / c]$. Since $M_{b} \geq M_{a^{*}}$, a point $t_{c} \in[0,1]$ exists such that $F_{b}\left(t_{c}\right) \geq M_{a^{*}}=F_{a^{*}}(1)$. Hence $t_{c} \in(1 / c, 1]$. On the other hand,

$$
F_{a^{*}}(1) \leq F_{b}\left(t_{c}\right)=c^{-2 H} F_{a^{*}}\left(c t_{c}\right) \leq\left(c t_{c}\right)^{-2 H} F_{a^{*}}\left(c t_{c}\right) .
$$


Since $c t_{c} \rightarrow 1+$ as $c \rightarrow 1+$, we get

$$
\left.\left(x^{-2 H} F_{a^{*}}(x)\right)_{x}^{\prime}\right|_{x=1} \geq 0
$$

and this is equivalent to the statement of the lemma.

The latter lemma implies, in particular, that $F_{a^{*}}^{\prime}(1)>0$, since $F_{a^{*}}(1)=M_{a^{*}}>0$.

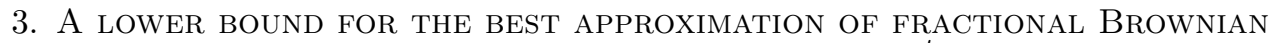
MOTION BY MARTINGALES OF THE FORM $\int_{0}^{t} a(s) d W_{s}$

Theorem 3.1. The square of the distance

$$
\rho_{T}:=\min _{a \in L_{2}[0, T]} \max _{0 \leq t \leq T} \mathrm{E}\left(B_{t}^{H}-\int_{0}^{t} a(s) d W_{s}\right)^{2}
$$

admits the following estimate:

$$
\rho_{T} \geq \max _{0 \leq t_{1} \leq 1} \frac{\left(1-t_{1}^{2 H}-\left(1-t_{1}\right)^{2 H}\right)^{2}}{16 t_{1}^{2 H}} \cdot T^{2 H}>0 .
$$

Proof. In view of property (2.1), $\rho_{T}$ can be rewritten via $\rho_{1}$, namely $\rho_{T}=T^{2 H} \cdot \rho_{1}$. This implies that one can restrict the consideration to the case of $\rho_{T}$ with $T=1$. Now we construct a lower bound for

$$
\max _{0 \leq t \leq 1} \mathrm{E}\left(B_{t}^{H}-\int_{0}^{t} a(s) d W_{s}\right)^{2}=\max _{0 \leq t \leq 1} \int_{0}^{t}(z(t, s)-a(s))^{2} d s .
$$

Let $0<t_{1} \leq 1$. Consider the random variable $\int_{0}^{1} a(s) d W_{s}=: B$. Then

$$
\begin{aligned}
\max _{0 \leq t \leq 1} \mathrm{E}\left(B_{t}^{H}-\int_{0}^{t} a(s) d W_{s}\right)^{2} \\
=\max _{0 \leq t \leq 1} \mathrm{E}\left(B_{t}^{H}-\mathrm{E}\left[B \mid \mathcal{F}_{t}\right]\right)^{2} \\
\geq \max \left(\mathrm{E}\left(B_{t_{1}}^{H}-\mathrm{E}\left[B \mid \mathcal{F}_{t_{1}}\right]\right)^{2}, \mathrm{E}\left(B_{1}^{H}-B\right)^{2}\right) \\
\geq \max \left(\mathrm{E}\left(B_{t_{1}}^{H}-\mathrm{E}\left[B \mid B_{t_{1}}^{H}\right]\right)^{2}, \mathrm{E}\left(\mathrm{E}\left[B_{1}^{H} \mid B_{t_{1}}^{H}\right]-\mathrm{E}\left[B \mid B_{t_{1}}^{H}\right]\right)^{2}\right) \\
\geq \frac{1}{2}\left(\mathrm{E}\left(B_{t_{1}}^{H}-\mathrm{E}\left[B \mid B_{t_{1}}^{H}\right]\right)^{2}+\mathrm{E}\left(\mathrm{E}\left[B_{1}^{H} \mid B_{t_{1}}^{H}\right]-\mathrm{E}\left[B \mid B_{t_{1}}^{H}\right]\right)^{2}\right) \\
\geq \frac{1}{4} \mathrm{E}\left(B_{t_{1}}^{H}-\mathrm{E}\left[B_{1}^{H} \mid B_{t_{1}}^{H}\right]\right)^{2} \\
=\frac{1}{4} \mathrm{E}\left(B_{t_{1}}^{H}-\frac{\mathrm{E}\left(B_{1}^{H} B_{t_{1}}^{H}\right)}{\mathrm{E}\left(\left(B_{t_{1}}^{H}\right)^{2}\right)} B_{t_{1}}^{H}\right)^{2}=\frac{1}{4} \mathrm{E}\left(B_{t_{1}}^{H} \cdot\left(1-\frac{1+t_{1}^{2 H}-\left(1-t_{1}\right)^{2 H}}{2 t_{1}^{2 H}}\right)\right)^{2} \\
=\frac{1}{4} t_{1}^{2 H}\left(1-\frac{1+t_{1}^{2 H}-\left(1-t_{1}\right)^{2 H}}{2 t_{1}^{2 H}}\right)^{2}=\frac{\left(1-t_{1}^{2 H}-\left(1-t_{1}\right)^{2 H}\right)^{2}}{16 t_{1}^{2 H}} .
\end{aligned}
$$

The theorem is proved.

Remark 3.2. Using the inequality $\frac{1}{2}\left(a^{2}+b^{2}\right) \geq\left(\frac{a+b}{2}\right)^{2}$, we obtain

$$
\frac{t_{1}^{2 H}+\left(1-t_{1}\right)^{2 H}}{2} \geq\left(\frac{t_{1}+\left(1-t_{1}\right)}{2}\right)^{2 H}=\frac{1}{2^{2 H}} \text {. }
$$


Moreover, the equality holds if and only if $t_{1}=1-t_{1}$, that is, if $t_{1}=\frac{1}{2}$. Thus

$$
\rho_{T} \geq \frac{\left(2^{2 H}-2\right)^{2}}{16 \cdot 2^{2 H}} \cdot T^{2 H}
$$

if $t_{1}=\frac{1}{2}$ in Theorem 3.1

\section{A COMPARISON OF THE BEST APPROXIMATIONS}

OF FRACTIONAL BROWNIAN MOTION IN SOME CLASSES OF FUNCTIONS

Let $\left\{B_{t}^{H}, t \geq 0\right\}$ be a fractional Brownian motion with the Hurst index $H \in\left(\frac{1}{2}, 1\right)$, $T>0$ be fixed, $a:[0, T] \rightarrow \mathbb{R}$ be a measurable function such that $a \in L_{2}[0, T]$, a square integrable martingale $\left\{M_{t}, t \in[0, T]\right\}$ be of the form $M_{t}=\int_{0}^{t} a(s) d W_{s}$, where $\left\{W_{t}, t \in[0, T]\right\}$ is a Wiener process related to the fractional Brownian motion $B^{H}$ as follows: for all $t \in[0, T]$,

$$
B_{t}^{H}=\int_{0}^{t} z(t, s) d W_{s}
$$

where

$$
z(t, s)=c_{H}(H-1 / 2) s^{1 / 2-H} \int_{s}^{t} u^{H-1 / 2}(u-s)^{H-3 / 2} d u .
$$

Representation (4.1) is proved in the paper [7]. Here

$$
c_{H}=\left(\frac{2 H \cdot \Gamma\left(\frac{3}{2}-H\right)}{\Gamma\left(H+\frac{1}{2}\right) \Gamma(2-2 H)}\right)^{1 / 2} .
$$

Class 5) in the list given in Section 1 is wider than the class of constant functions; namely class 5) consists of the functions $a(s)=k_{1}+k_{2} \cdot s^{\alpha}, s \in[0, T]$, for $k_{1}, k_{2} \in \mathbb{R}$. Thus the best approximation by the elements of this class is not worse than that by elements of the class 1), that is,

$$
\min _{k} \max _{0 \leq t \leq T} \mathrm{E}\left(B_{t}^{H}-\int_{0}^{t} k d W_{s}\right)^{2} \geq \min _{k_{1}, k_{2}} \max _{0 \leq t \leq T} \mathrm{E}\left(B_{t}^{H}-\int_{0}^{t}\left(k_{1}+k_{2} s^{\alpha}\right) d W_{s}\right)^{2} .
$$

We prove that the inequality is strict; in fact,

$$
\min _{k} \max _{0 \leq t \leq T} \mathrm{E}\left(B_{t}^{H}-k W_{t}\right)^{2}>\min _{k_{1}, k_{2}} \max _{0 \leq t \leq T} \mathrm{E}\left(B_{t}^{H}-\int_{0}^{t}\left(k_{1}+k_{2} s^{\alpha}\right) d W_{s}\right)^{2} .
$$

Put $f\left(t, k_{1}, k_{2}\right):=\mathrm{E}\left(B_{t}^{H}-\int_{0}^{t}\left(k_{1}+k_{2} s^{\alpha}\right) d W_{s}\right)^{2}$.

It is known that $k_{2}^{0}<0$ and $k_{2}^{*}<0$. Note that $f\left(T, k_{1}, k_{2}\right)$ is a polynomial of the second order of variables $k_{1}$ and $k_{2}$; moreover, the polynomial is concave with respect to $k_{1}$ and $k_{2}$ and attains its minimum at the point $\left(k_{1}^{*}, k_{2}^{*}\right)$. For $k_{2} \geq k_{2}^{0}$, the function $f\left(t, k_{1}, k_{2}\right)$ increases with respect to $t$ in the interval $[0,+\infty)$. It is proved in 3 ] that

$$
\min _{k} \max _{0 \leq t \leq T} f(t, k, 0)=T^{2 H}\left(1-c_{1}^{2}(H)\right),
$$

where

$$
c_{1}(H)=\frac{\alpha}{\alpha+1} c_{H} B(1-\alpha, \alpha)
$$

The minimum is attained at $k=c_{1}(H) \cdot T^{\alpha}$, while the maximum

$$
\max _{0 \leq t \leq T} f\left(t, c_{1}(H) T^{\alpha}, 0\right)
$$

is attained at $t=T$. 
We have $\left(c_{1}(H) T^{\alpha}, 0\right) \neq\left(k_{1}^{*}, k_{2}^{*}\right)$, since $k_{2}^{*}<0$. Thus $f\left(T, k_{1}, k_{2}\right)$ does not have a local minimum at the point $\left(c_{1}(H) T^{\alpha}, 0\right)$. This implies that there exists a point $\left(k_{1}^{(2)}, k_{2}^{(2)}\right)$, $k_{2}^{(2)}>k_{2}^{0}$, such that $f\left(T, c_{1}(H) T^{\alpha}, 0\right)>f\left(T, k_{1}^{(2)}, k_{2}^{(2)}\right)$.

The minimum of (4.3) is attained at $k=c_{1}(H) \cdot T^{\alpha}$. Taking into account the inequality $k_{2}^{(2)}>k_{2}^{0}$ and that the function $f\left(t, k_{1}^{(2)}, k_{2}^{(2)}\right)$ increases, we obtain the following bound:

$$
\begin{aligned}
\min _{k} \max _{0 \leq t \leq T} f(t, k, 0) & =f\left(T, c_{1}(H) T^{\alpha}, 0\right)>f\left(T, k_{1}^{(2)}, k_{2}^{(2)}\right)=\max _{0 \leq t \leq T} f\left(t, k_{1}^{(2)}, k_{2}^{(2)}\right) \\
& \geq \min _{k_{1}, k_{2}} \max _{0 \leq t \leq T} f\left(t, k_{1}, k_{2}\right) .
\end{aligned}
$$

To show that the best approximation by functions of the class 5 ) is better than that by functions of the class 1 ), we need to prove that

$$
\begin{aligned}
T^{2 \alpha+1} & \left(1-\left(\frac{\alpha c_{H}}{\alpha+1} \cdot B(1-\alpha, \alpha)\right)^{2}\right) \\
> & T^{2 \alpha+1}\left(1-c_{H}^{2}\left(B^{2}(1-\alpha, \alpha)-\frac{2 B(1-\alpha, \alpha)}{\alpha}+\frac{(\alpha+1)^{2}}{\alpha^{2}(2 \alpha+1)}\right)\right)
\end{aligned}
$$

or, in other words,

$$
B^{2}(1-\alpha, \alpha)-\frac{2 B(1-\alpha, \alpha)}{\alpha}+\frac{(\alpha+1)^{2}}{\alpha^{2}(2 \alpha+1)}>\left(\frac{\alpha c_{H}}{\alpha+1} \cdot B(1-\alpha, \alpha)\right)^{2} .
$$

Consider the difference between the left and right hand sides of (4.4):

$$
\begin{aligned}
B^{2}(1 & -\alpha, \alpha)-\frac{2 B(1-\alpha, \alpha)}{\alpha}+\frac{(\alpha+1)^{2}}{\alpha^{2}(2 \alpha+1)}-\left(\frac{\alpha}{\alpha+1} \cdot B(1-\alpha, \alpha)\right)^{2} \\
& =\frac{2 \alpha+1}{(\alpha+1)^{2}} B^{2}(1-\alpha, \alpha)-\frac{2 B(1-\alpha, \alpha)}{\alpha}+\frac{(\alpha+1)^{2}}{\alpha^{2}(2 \alpha+1)} \\
& =(2 \alpha+1)\left(\frac{B(1-\alpha, \alpha)}{\alpha+1}-\frac{\alpha+1}{\alpha(2 \alpha+1)}\right)^{2} \geq 0
\end{aligned}
$$

Now we show that the right hand side of (4.5) does not equal zero, that is,

$$
f(\alpha):=(\alpha+1)^{2} \cdot \sin \pi \alpha-\pi \alpha(2 \alpha+1) \neq 0 .
$$

In fact, we will prove that $f(\alpha)<0$ for $0<\alpha<\frac{1}{2}$.

We use the Taylor expansion with a remainder term written in the Lagrange form:

$$
f(\alpha)=f(0)+\alpha f^{\prime}(0)+\frac{\alpha^{2} f^{\prime \prime}(0)}{2}+\frac{\alpha^{3} f^{\prime \prime \prime}(\theta \alpha)}{3 !}, \quad 0<\theta<1 .
$$

It is obvious that $f^{\prime}(0)=0, f^{\prime \prime}(0)=0$, and $f^{\prime \prime \prime}(\theta \alpha)<0$. Indeed,

$$
f^{\prime \prime \prime}(\alpha)=\pi \cos \pi \alpha\left(6-(\alpha+1)^{2} \pi^{2}\right)-6(\alpha+1) \pi^{2} \sin \pi \alpha<0 .
$$

Since $\alpha^{3} f^{\prime \prime \prime}(\theta \alpha) / 3 !<0$, we obtain $f(\alpha)<0$ for $0<\alpha<\frac{1}{2}$.

This proves that (4.4) holds, indeed.

5. An upper Bound For the SquARE OF THE Distance $\rho_{T}$ IN THE Class of FUNCTIONS $a(s)=a_{0} s^{\alpha}+a_{1} s^{\alpha+1}$

Let $M_{t}=\int_{0}^{t} a(s) d W_{s}, B_{t}^{H}=\int_{0}^{s} z(t, s) d W_{s}$, and let

$$
z(t, s)=c_{H} \alpha s^{-\alpha} \int_{s}^{t} u^{\alpha}(u-s)^{\alpha-1} d u .
$$


We apply Theorem 2 of the paper [3] and obtain

$$
\int_{0}^{t}\left(z(t, s)-c_{H} s^{\alpha}\right)^{2} d s=t^{2 H}\left(1-\frac{c_{H}^{2}}{2 H}\right) .
$$

According to this result,

$$
\begin{aligned}
\mathrm{E}\left(B_{t}^{H}-M_{t}\right)^{2}= & \int_{0}^{t}(z(t, s)-a(s))^{2} d s \\
= & \int_{0}^{t}\left(\left(z(t, s)-c_{H} s^{\alpha}\right)+\left(c_{H} s^{\alpha}-a(s)\right)\right)^{2} d s \\
= & \int_{0}^{t}\left(z(t, s)-c_{H} s^{\alpha}\right)^{2} d s \\
& +2 \int_{0}^{t}\left(z(t, s)-c_{H} s^{\alpha}\right) \cdot\left(c_{H} s^{\alpha}-a(s)\right) d s \\
& +\int_{0}^{t}\left(c_{H} s^{\alpha}-a(s)\right)^{2} d s \\
= & t^{2 H}\left(1-\frac{c_{H}^{2}}{2 H}\right) \\
& -2 \alpha c_{H} \int_{0}^{t} u^{\alpha} \int_{0}^{u}(u-s)^{\alpha-1} \cdot\left(a(s) s^{-\alpha}-a(u) u^{-\alpha}\right) d s d u \\
& +\int_{0}^{t}\left(c_{H} s^{\alpha}-a(s)\right)^{2} d s .
\end{aligned}
$$

Making the change of variables $b(u)=a(u) u^{-\alpha}$ and $c(u)=b(u)-c_{H}$ we obtain $(5.2)$

$$
\begin{aligned}
\mathrm{E}\left(B_{t}^{H}-M_{t}\right)^{2}= & t^{2 H}\left(1-\frac{c_{H}^{2}}{2 H}\right)-2 \alpha c_{H} \int_{0}^{t} u^{\alpha} \int_{0}^{u}(u-s)^{\alpha-1} \cdot(b(s)-b(u)) d s d u \\
& +\int_{0}^{t} s^{2 \alpha}\left(b(s)-c_{H}\right)^{2} d s \\
= & t^{2 H}\left(1-\frac{c_{H}^{2}}{2 H}\right)-2 \alpha c_{H} \int_{0}^{t} u^{\alpha} \int_{0}^{u}(u-s)^{\alpha-1} \cdot(c(s)-c(u)) d s d u \\
& +\int_{0}^{t} s^{2 \alpha} c^{2}(s) d s
\end{aligned}
$$

Put $c(u)=a_{0}+a_{1} u$ on the right hand side of (5.2), which means that

$$
a(u)=\left(a_{0}+c_{H}+a_{1} u\right) u^{\alpha}
$$

in (5.1). The optimization with respect to both coefficients $a_{0}$ and $a_{1}$ requires a cumbersome calculation; thus we minimize the distance by using Lemmas 2.1 and 2.2. In doing so, we consider the following class of functions:

$$
\mathcal{A}=\left\{a \in L_{2}[0,1]: F_{a}^{\prime}(1)=2 H F_{a}(1)\right\} .
$$

Theorem 5.1. Let $\frac{1}{2}<H<1$ and $\alpha=H-\frac{1}{2}$. Then the minimum

$$
\min _{a_{0}, a_{1}} \max _{0 \leq t \leq T} \mathrm{E}\left(B_{t}^{H}-\int_{0}^{t}\left(a_{0} s^{\alpha}+a_{1} s^{\alpha+1}\right) d W_{s}\right)^{2}
$$


in the class of functions $c(u)=a_{0}+a_{1} u \in \mathcal{A}$ is attained for the coefficients $a_{0}=a_{0}^{*}$ and $a_{1}=a_{1}^{*}$, where

$$
\begin{gathered}
a_{0}^{*}=\frac{\alpha(2 \alpha+1)(2 \alpha+3)}{(\alpha+1)} c_{H}, \\
a_{1}^{*}=-\frac{2 \alpha+3}{T(2 \alpha+2)} \cdot\left(a_{0}+\frac{\alpha c_{H}}{\alpha+1}\right) .
\end{gathered}
$$

The minimum is equal to

$$
T^{2 \alpha+1}\left[1-\frac{c_{H}^{2}}{2 \alpha+1}-\frac{\alpha^{2}(2 \alpha+3) c_{H}^{2}}{(\alpha+1)^{2}}\right] .
$$

Proof. Substituting $c(u)=a_{0}+a_{1} u$ in (5.2) we obtain

$$
\begin{aligned}
F_{a}(t)= & \mathrm{E}\left(B_{t}^{H}-M_{t}\right)^{2} \\
= & t^{2 H}\left(1-\frac{c_{H}^{2}}{2 H}\right)+2 \alpha c_{H} \int_{0}^{t} u^{\alpha} \int_{0}^{u}(u-s)^{\alpha} \cdot a_{1} d s d u \\
& \quad+\int_{0}^{t} s^{2 \alpha}\left(a_{0}^{2}+2 a_{0} a_{1} s+a_{1}^{2} s^{2}\right) d s \\
= & t^{2 \alpha+1}\left(1-\frac{c_{H}^{2}}{2 \alpha+1}+\frac{a_{0}^{2}}{2 \alpha+1}\right)+t^{2 \alpha+2} \frac{a_{1}}{\alpha+1}\left(a_{0}+\frac{\alpha c_{H}}{\alpha+1}\right) \\
& \quad+t^{2 \alpha+3} \cdot \frac{a_{1}^{2}}{2 \alpha+3} .
\end{aligned}
$$

Passing to the derivatives with respect to $t$ on both sides of (5.3) we get

$$
F_{a}^{\prime}(t)=t^{2 \alpha}\left(\kappa_{0}+\kappa_{1} t+\kappa_{2} t^{2}\right)
$$

where

$$
\kappa_{0}=2 \alpha+1-c_{H}^{2}+a_{0}^{2}, \quad \kappa_{1}=2 a_{1}\left(a_{0}+\frac{\alpha c_{H}}{\alpha+1}\right), \quad \kappa_{2}=a_{1}^{2} .
$$

Recalling that the functions under consideration belong to the class $\mathcal{A}$, we evaluate at the point $T$ that

$$
T^{2 \alpha}\left(\kappa_{0}+\kappa_{1} T+\kappa_{2} T^{2}\right)=\frac{2 \alpha+1}{T} \cdot T^{2 \alpha+1} \cdot\left(\frac{\kappa_{0}}{2 \alpha+1}+T \cdot \frac{\kappa_{1}}{2 \alpha+2}+T^{2} \cdot \frac{\kappa_{2}}{2 \alpha+3}\right),
$$

whence

$$
\kappa_{2}=-\kappa_{1} \cdot \frac{2 \alpha+3}{2 T \cdot(2 \alpha+2)} .
$$

The latter equality is equivalent to the following one:

$$
a_{1}=-\frac{2 \alpha+3}{T \cdot(2 \alpha+2)} \cdot\left(a_{0}+\frac{\alpha c_{H}}{\alpha+1}\right),
$$

whence

$$
\begin{aligned}
F_{a}(T)=T^{2 \alpha+1}[ & {\left[1-\frac{c_{H}^{2}}{2 \alpha+1}\right.} \\
& \left.+\frac{1}{(2 \alpha+2)^{2}}\left(a_{0}^{2} \cdot \frac{1}{2 \alpha+1}-a_{0} \cdot \frac{2(2 \alpha+3) \alpha c_{H}}{\alpha+1}-\frac{(2 \alpha+3) \alpha^{2} \cdot c_{H}^{2}}{(\alpha+1)^{2}}\right)\right] .
\end{aligned}
$$


The minimum of the expression on the right hand side of (5.8) with respect to $a_{0}$ is attained at

$$
a_{0}=\frac{\alpha(2 \alpha+1)(2 \alpha+3)}{(\alpha+1)} \cdot c_{H}
$$

and is equal to

$$
\begin{aligned}
\min _{a_{0}} F_{a}(T) & =T^{2 \alpha+1}\left[1-\frac{c_{H}^{2}}{2 \alpha+1}+\frac{1}{(2 \alpha+2)^{2}}\left(-\frac{\alpha^{2}(2 \alpha+3)^{2}(2 \alpha+1) c_{H}^{2}}{(\alpha+1)^{2}}\right.\right. \\
& =T^{2 \alpha+1}\left[1-\frac{c_{H}^{2}}{2 \alpha+1}-\frac{\alpha^{2}(2 \alpha+3) c_{H}^{2}}{(\alpha+1)^{2}}\right] .
\end{aligned}
$$

It remains to prove that the function $F_{a}(t)$ increases with respect to $t$ if $a_{0}=a_{0}^{*}$ and $a_{1}=a_{1}^{*}$. Then

$$
\min _{a_{0}, a_{1}} \max _{0 \leq t \leq T} F_{a}(T) \leq F_{a^{*}}(T), \quad a^{*}=\left(a_{0}^{*}, a_{1}^{*}\right) .
$$

Taking into account (5.4), we need to prove that the least root of the equation

$$
\kappa_{0}+\kappa_{1} t+\kappa_{2} t^{2}=0
$$

exceeds $T$. Without loss of generality we restrict the consideration to the case of $T=1$. Then this root is equal to

$$
t_{1}=\frac{-\kappa_{1}-\sqrt{\kappa_{1}^{2}-4 \kappa_{0} \kappa_{2}}}{2 \kappa_{2}}=\alpha+1-\frac{1}{\alpha c_{H}} \sqrt{c_{H}^{2} \alpha^{2}(\alpha+1)^{2}-\kappa_{0}} .
$$

The inequality $t_{1}>1$ is equivalent to

$$
\alpha^{2}>(\alpha+1)^{2}-\frac{\kappa_{0}}{\alpha^{2} c_{H}^{2}} \quad \text { or } \quad 2 \alpha+1<\frac{\kappa_{0}}{\alpha^{2} c_{H}^{2}} .
$$

On the other hand,

$$
\begin{aligned}
\frac{\kappa_{0}}{\alpha^{2} c_{H}^{2}} & =\left(2 H-c_{H}^{2}+\frac{\alpha^{2} c_{H}^{2}(2 \alpha+1)^{2}(2 \alpha+3)^{2}}{(\alpha+1)^{2}}\right) \cdot \frac{1}{\alpha^{2} c_{H}^{2}} \\
& =\frac{2 H-c_{H}^{2}}{(\alpha+1)^{2}}+\frac{(2 \alpha+1)^{2}(2 \alpha+3)^{2}}{(\alpha+1)^{2}}>\frac{(2 \alpha+1)^{2}(2 \alpha+3)^{2}}{(\alpha+1)^{2}}>(2 \alpha+1)^{2}>2 \alpha+1,
\end{aligned}
$$

that is, $t_{1}>1$, indeed.

Corollary 5.2. Equality (5.9) implies

$$
1-\frac{c_{H}^{2}}{2 \alpha+1}-\frac{\alpha^{2}(2 \alpha+3) c_{H}^{2}}{(\alpha+1)^{2}}>0
$$

whence we obtain a bound for $c_{H}$ :

$$
c_{H}<\sqrt{\frac{(2 \alpha+1)(\alpha+1)^{2}}{(\alpha+1)^{2}+\alpha^{2}(2 \alpha+1)(2 \alpha+3)}}=: C_{H}^{(c)} .
$$

Estimate (5.10) obviously improves the estimate $c_{H}^{2} \leq 2 H$ obtained in the paper [2]. 


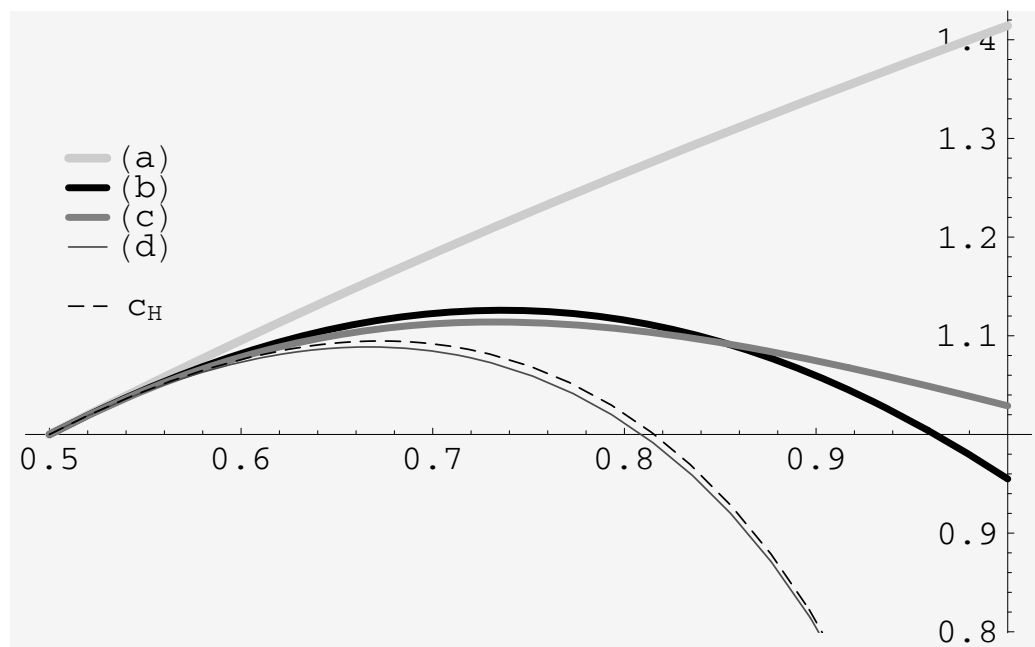

FiguRE 1

\section{A COMPARISON OF SOME LOWER AND UPPER BOUNDS FOR $c_{H}$}

Several upper bounds for the constant $c_{H}$ are obtained in the papers $[2]$ and $[3$. Below are two of these bounds:

$$
c_{H}<\sqrt{2 \alpha+1}=: C_{H}^{(a)}
$$

([2], Corollary 1; Figure 1, graph (a));

$$
c_{H}<\frac{(\alpha+1) \sin \pi \alpha}{\pi \alpha}=: C_{H}^{(b)}
$$

([3], Remark 1; Figure 1, graph (b)). The latter bound is obtained by transforming the distance

$$
\rho_{T}=T^{2 H}\left(1-c_{1}^{2}\right)
$$

where

$$
c_{1}=c_{1}(H)=\alpha c_{H} \cdot \frac{1}{\alpha+1} \cdot B(1-\alpha, \alpha) .
$$

Relation (5.10) provides another upper bound for $c_{H}$, namely $C_{H}^{(c)}$.

The paper 4] (Lemma 3.1; Figure 1, graph (d)) contains a lower bound for the constant $c_{H}$, namely

$$
c_{H}>\sqrt{8 H^{2}(1-H)}=: C_{H}^{(d)} .
$$

Below we compare these four bounds with the help of Mathematica.

It is seen from Figure 1 that $C_{H}^{(b)}$ approximates $c_{H}$ better than $C_{H}^{(a)}$ does in the whole interval $\left(\frac{1}{2}, 1\right)$. It is also clear that $C_{H}^{(c)}$ approximates $c_{H}$ better than $C_{H}^{(b)}$ does in the interval $H \in\left(\frac{1}{2}, 0.853497\right)$ (the upper end point of this interval is found numerically with the help of Mathematica). On the other hand, $C_{H}^{(b)}$ approximates $c_{H}$ better than $C_{H}^{(c)}$ does in the interval $H \in(0.853497,1)$. The graphs of the differences $C_{H}^{(a)}-C_{H}^{(b)}$ and $C_{H}^{(b)}-C_{H}^{(c)}$ depicted in Figure 2 and Figure 3 confirm this conclusion. 


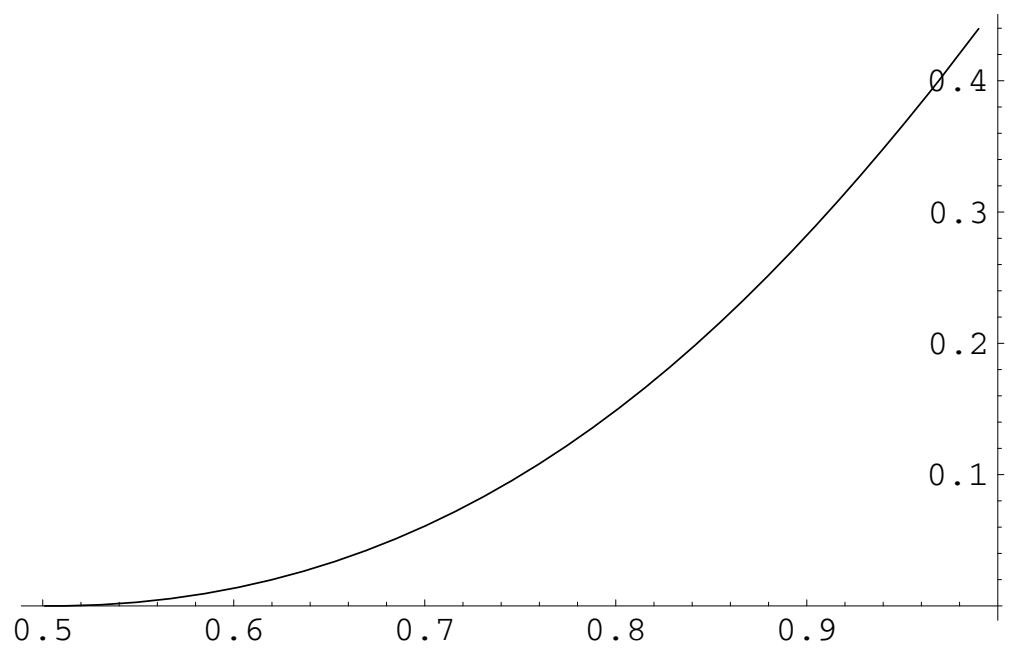

FIGURE 2

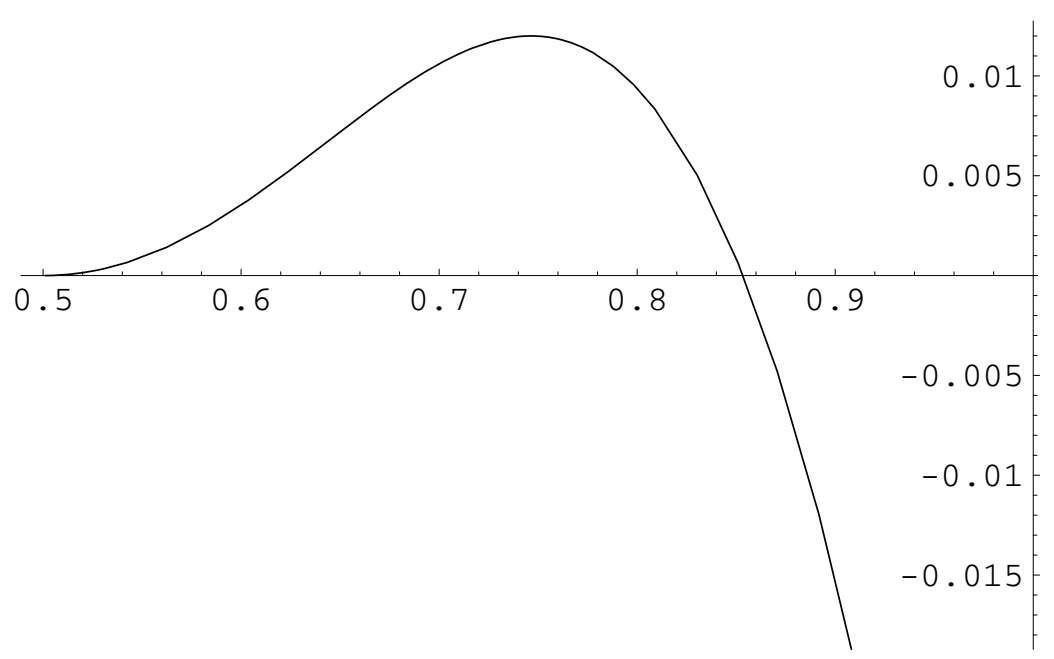

FIGURE 3

\section{Concluding Remarks}

We obtained some upper bounds for the constant $c_{H}$. The bounds $C_{H}^{(b)}$ and $C_{H}^{(c)}$ are the best among them in the corresponding intervals mentioned above. This allows one to determine the best upper bound for $\rho_{T}$.

We compare the upper bound for $\rho_{T}$ and lower bound (3.1) (see Figure 4, curve (a)). We substitute the expression for $c_{H}$ given by (1.1) to (6.3) and to (5.9) (see Figure 4, curve (c) and Figure 4, curve (b)). Thus we obtain the best upper bounds for $\rho_{T}$. The difference between the curves (b) and (c) presented on Figure 4 is depicted on Figure 5. We see that the best upper bound for $\rho_{T}$ is given by the approximation obtained from (5.9) in the interval $\left(\frac{1}{2}, 0.720536\right)$ and by the approximation obtained from (6.3) in the interval $(0.720536,1)$. 


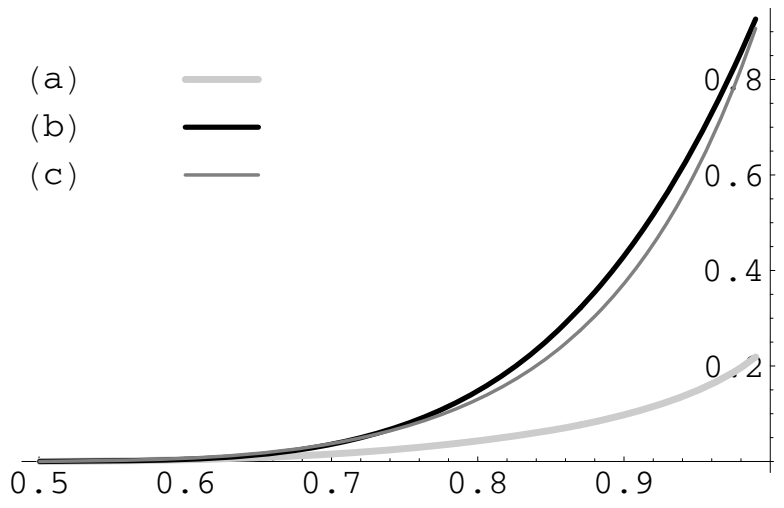

Figure 4

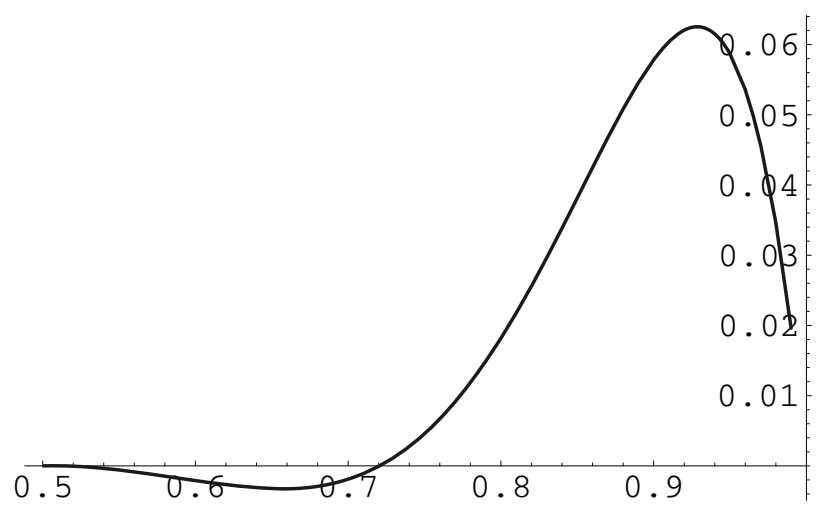

FiguRE 5

\section{BIBLIOGRAPHY}

1. T. O. Androshchuk, Approximation of a stochastic integral with respect to fractional Brownian motion by integrals with respect to absolutely continuous processes, Teor. Imovir. Mat. Stat. 73 (2005), 17-26; English transl. in Theory Probab. Math. Statist. 73 (2006), 19-29. MR2213333 (2006m:60072)

2. O. L. Banna, An approximation of the fractional Brownian motion whose Hurst index is near the unity by stochastic integrals with linear-power integrands, Applied Statistics. Actuarial and Finance Mathematics 1 (2007), 60-67. (Ukrainian)

3. O. L. Banna and Yu. S. Mishura, The simplest martingales for the approximation of the fractional Brownian motion, Visnyk Kyiv National Taras Shevchenko University. Mathematics and Mechanics 19 (2008), 38-43. (Ukrainian)

4. Yu. S. Mishura and O. L. Banna, Approximation of fractional Brownian motion by Wiener integrals, Teor. Imovir. Mat. Stat. 79 (2008), 96-104; English transl. in Theory Probab. Math. Statist. 79 (2009), 107-116. MR2494540(2010b:60113)

5. T. Androshchuk and Y. S. Mishura, Mixed Brownian-fractional Brownian model: absence of arbitrage and related topics, Stochastics 78 (2006), 281-300. MR2270939 (2007k:60198)

6. O. Banna and Y. S. Mishura, Approximation of fractional Brownian motion with associated Hurst index separated from 1 by stochastic integrals of linear power functions, Theory Stoch. Processes 14(30) (2008), no. 3-4, 1-16. MR2498600 (2010d:60099) 
7. I. Norros, E. Valkeila, and J. Virtamo, An elementary approach to a Girsanov formula and other analytical results on fractional Brownian motions, Bernoulli 5(4) (1999), 571-587. MR.1704556 (2000f:60053)

8. T. H. Thao, A note on fractional Brownian motion, Vietnam J. Math. 31 (2003), no. 3, 255260. MR2010525(2004j:60081)

Department of Probability Theory, Statistics, and Actuarial Mathematics, Faculty for Mechanics and Mathematics, National Taras Shevchenko University, Academician Glushkov Avenue 2, Kiev 03127, Ukraine

E-mail address: bannaya@mail.univ.kiev.ua

Department of Probability Theory, Statistics, and Actuarial Mathematics, Faculty for Mechanics and Mathematics, National Taras Shevchenko University, Academician Glushkov Avenue 2, Kiev 03127, Ukraine

E-mail address: myus@univ.kiev.ua

Received 7/APR/2010

Translated by N. SEMENOV 